\title{
ESTIMATIONS OF LOCAL MAGNETIC FIELDS IN SOLAR FLARES: BASIC METHODS AND SOME RESULTS
}

\author{
V.G. Lozitsky ${ }^{1}$, E.A. Baranovsky ${ }^{2}$, N.I. Lozitska ${ }^{1}$, V.P.Tarashchuk ${ }^{2}$ \\ ${ }^{1}$ Astronomical Observatory of the Taras Shevchenko National University \\ of Kyiv, Kyiv, Ukraine,lozitsky@observ.univ.kiev.ua,nloz@observ.univ.kiev.ua \\ ${ }^{2}$ Crimea Astrophysical Observatory, Nauchny, Crimea \\ edward@craocrimea.ru,veratar4@gmail.com
}

\begin{abstract}
We consider three methods for estimations of local magnetic fields in solar flares: (1) analysis of bisectors of $I \pm V$ profiles (Lozitsky, 2015); (2) search for Zeeman-like effects in cores of spectral lines with very low Lande factors, $g_{\text {eff }} \approx 0.01$ (Lozitsky, 1993, 1998); (3) semi-empirical modeling using many spectral lines and two-component models (see, e.g., Lozitsky et al., 2000). We illustrate the application of named methods to different observational data and to different spectral lines. Our main conclusions are following: (a) upper limit of local magnetic fields in solar flares is, at least, $\sim 10^{4} \mathrm{G}$, (b) such extremely strong fields can occur in very small, spatially unresolved scales, (c) lifetime of such fields is, at least, a few minutes.
\end{abstract}

Keywords: Sun, solar activity, solar flares, magnetic fields, spectral lines, the Zeeman effect, diagnostics of spatially unresolved structures, extremely strong fields.

\section{Introduction}

Solar magnetic fields have very fine structure, with size of smallest elements under the instrumental limit for modern solar telescopes. In fact, this limit is on level of 50-70 $\mathrm{km}$ at present (see, e.g., Varsik et al., 2014) whereas the smallest magnetic elements on the Sun, likely, have the size about 15-20 km (Stenflo, 2011, Botygna et al., 2016). Due to this reason, direct magnetic observations allow to obtain some average (effective) magnetic field $B_{\text {eff }}$ which can be very differ from local magnetic field $B_{\text {loc }}$. For nonflare regions, $B_{\text {loc }}$ is of kilogauss range (see, e.g. Stenflo, 1973; Rachkovsky et al., 2005) whereas $B_{\text {eff }}$ can be 1-3 orders weaker, and it reflects, in general, the magnetic flux rather than true local field.

The diagnostics of spatially unresolved magnetic fields in solar flares is more complicated than in unperturbed regions outside flares. For non-flare regions, one can apply the line ratio method or its modification (see, e.g., Stenflo, 1973, Rachkovsky et al., 2005). For flares, especially for wide emission line profiles, this method is not suitable. Therefore, it is necessary to use other methods, which are also quite informative, but are based on other approaches to the problem of estimating local fields. Below, three such methods will be considered, with a brief summary of the results obtained and their discussion.

\section{Analysis of bisectors of $I \pm V$ profiles}

In a general case, the diagnostics of spatially unresolved magnetic structures requires the application of at least two-component models for interpretation of observations. However, in this case number of free parameters is greatly increased (till about ten), which makes the final conclusions very vague, dependent on some simplifying assumptions. One can try to apply a simplified approach based on fixing and analyzing explicit deviations from the case of a weak one-component magnetic field. This is the main idea of the method for analyzing the bisectors of $I \pm V$ profiles of magnetosensitive spectral lines.

In weak-field approximation, Stokes $V$ parameter could be presented via formula

$$
V \propto(d I / d \lambda) \Delta \lambda_{\mathrm{H}}
$$

where $d I / d \lambda$ is Stokes $I$ gradient, $\Delta \lambda_{\mathrm{H}}-$ Zeeman splitting

$$
\Delta \lambda_{\mathrm{H}}=4.67 \times 10^{-13} g_{\mathrm{eff}} \lambda^{2} B,
$$

where $\Delta \lambda_{\mathrm{H}}$ and $\lambda$ are in $\AA$, and $B$ in gauss $(\mathrm{G}) ; g_{\text {eff }}-$ effective Lande factor. In fact, formula (1) follows from the condition that $I+V$ and $I-V$ profiles have analogous shape, but they are shifted mutually on $2 \Delta \lambda_{\mathrm{H}}$. This means that their bisectors should be parallel mutually, if $\Delta \lambda_{\mathrm{H}}<<$ $\Delta \lambda_{1 / 2}$, where $\Delta \lambda_{1 / 2}$ is half-width of a spectral line.

As to real observed profiles, bisectors are often not parallel even in case if $\Delta \lambda_{\mathrm{H}}<\Delta \lambda_{1 / 2}$. There are two main types of nonparallelism: (1) a monotonous increase in the splitting of bisectors as the line center is approached and (2) the non-monotonic course of this splitting, such as "loop" or "bulb". Both cases do not correspond to the approximation of a weak one-component magnetic field and require consideration in the framework of inhomogeneous magnetic fields. Figs. 1-3 present the examples of observations with non-parallelism of bisectors of (2) type. 
One can see that splitting of bisectors $\Delta \lambda_{\mathrm{B}}$ has a maximum on distances $\Delta \lambda_{\max } \approx 200-350 \mathrm{~m} \AA$. Let us remember, in weak-field approximation should be $\Delta \lambda_{B}=$ const.

Accoding to modelling data, similar spectral peculiarities are possible in two cases: (a) two-component model, with weak and strong fields, with quasi-gaussian profiles in both components, and very narrow spectral lines in second (spatially unresolved) component with strong field, (b) also two-component model, but with non-gaussian profiles in strong component. In (a) case, observed value $\Delta \lambda_{\max }$ corresponds, approximately, the Zeeman splitting $\Delta \lambda_{\mathrm{H}}$ in strong component (Gordovskyy and Lozitsky, 2014). This criterium gives very strong field in second component, in range of $\sim 10^{4} \mathrm{G}$. At heights of about 40 Mm (Fig. 2), such fields are unlikely.

Consideration of case (b) allows us to reduce the value of local fields in sub-telescopic structures. Namely, then the "loop" of bisectors appears not because of the large Zeeman splitting, but because of the non-Gaussian profiles with a flat top having a steep intensity gradient at a certain distance from the center. However, this requires the presence of a large optical thickness in sub-telescopic structures with an amplified field.

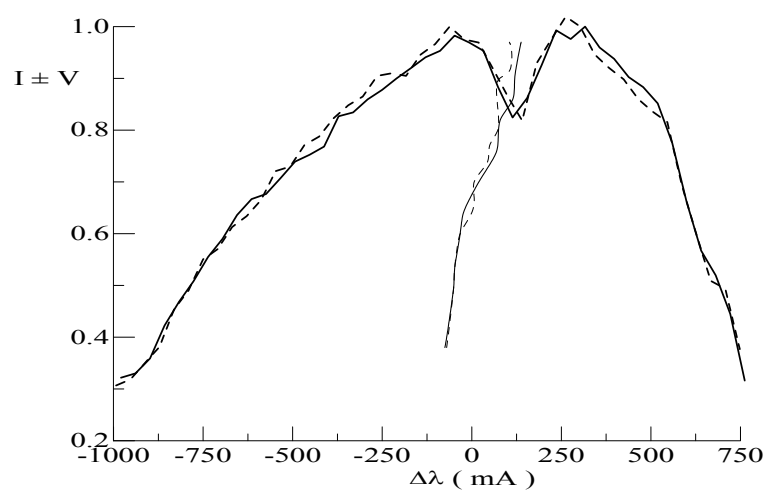

Figure 1: Observed $I+V$ and $I-V$ profiles and bisectors of H $\alpha$ line $\left(g_{\text {eff }}=1.05\right)$ in limb solar flare of 19 July 2012 of M 7.7 class (Kirichek et al., 2013).

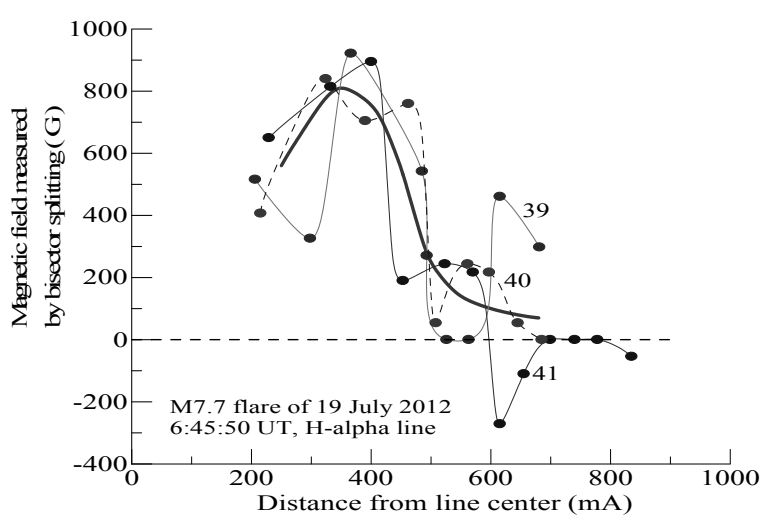

Figure 2: Observed splitting of bisectors of $I \pm V$ profiles versus distance from line center for limb solar flare of 19 July 2012 of M 7.7 class. Numbers 39-41 correspond to distance of the place under study from the solar limb (Kirichek et al., 2013).

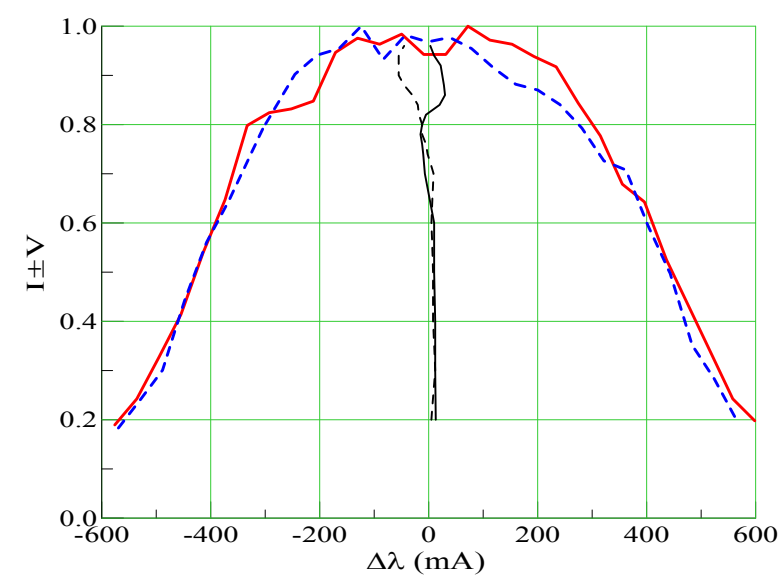

Figure 3: The same as on Fig. 1, but for quiet prominence of 09 November 2011 (Lozitsky et al., 2015).

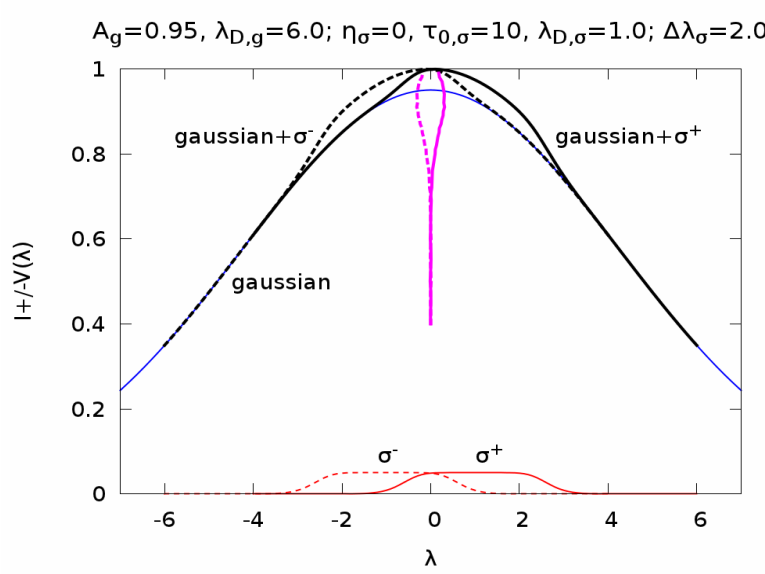

Figure 4: Theoretical $I \pm V$ profiles and their bisectors for two-component magnetic field (Lozitsky et al., 2015).

Fig. 4 illustrates case (b) with such parameters: the filling factor of the small-scale component is $5 \%$, and the optical thickness in it is 10 . The Doppler width in this component is 6 times smaller than in the background component that indicates the reduce of kinetic temperature. It was also believed that the magnetic field in the background component is zero, and the field in the smallscale component is purely longitudinal.

Comparing Figures 3 and 4, we see their good qualitative correspondence. If the horizontal scale of Figure 3 is attached to Figure 4, then the Zeeman splitting in the small-scale component should be approximately $100 \mathrm{~mA}$. This gives the magnitude of the magnetic field in the strong component of about $5 \mathrm{kG}$. Of course, this is also a very strong field for the level of the lower corona. Another thing is paradoxically here: the plasma density in regions with such a strong field should be substantially increased. Obviously, this poses an acute problem of the equilibrium of such structures: it is not clear what can compensate the huge magnetic and gas pressures inside the small-scale component. Perhaps there exists some specific topology of the magnetic field, for example, in the form of a vortex (Soloviev, 2013). Notice, earlier Kurochka and Tel'njukAdamchuk (1978), using data in unpolarized light, also came to the conclusion that the emission elements of the flare should be dense, optically opaque. 


\section{Spectral effects in lines with $g_{\text {eff }} \approx 0.01$}

A simple test for testing the hypothesis of the existence of especially strong magnetic fields is as follows. Spectral lines with very small Lande factors, about 0.01 , should not show signs of Zeeman effect if the signal-to-noise ratio for observations is about 50-100, and the magnetic field value does not exceed $5 \mathrm{kG}$. In the visible region of the solar spectrum, there are at least three iron lines, very convenient for such a check. These are FeI lines $\lambda=$ $5123.723,5434.527$ and $5576.099 \AA$... Theoretically, for $L S$ coupling, their effective Lande factors are $g_{\mathrm{LS}}=0.000$, but experimental values, determined in a laboratory, are $\mathrm{g}_{\mathrm{Lab}}=-0.013,-0.014$ and -0.012 , respectively (Landi Degl'Innocenti E.L.: 1982).

The first results of this kind were obtained by Lozitsky (1984) for the photosphere of the active region outside the spots. The photometric profiles were averaged over a large area on the Sun $(\approx 25 \mathrm{Mm})$ to increase the signal-to-noise ratio. It turned out that in the nuclei of lines 5123 and 5576 there is a weak splitting, which for their Lande factors should correspond to a magnetic field of about $3 \mathrm{kG}$.

For flares, a similar result was obtained nine years late (Lozitsky, 1993). It turned out that the emission peaks in lines FeI 5123.7 and FeI 5434.5 are also sometimes split, the value of the corresponding magnetic field reaching 90 $\mathrm{kG}$.

Till present, the splitting effect in the nuclei of lines with very low Lande factors (about 0.01) was found in six solar flares. Some examples of the observed effects are shown in Figs. 5-7.

Although in most cases the splitting effects mentioned above appear in the cores of the lines $(\Delta \lambda \leq 100 \mathrm{~m} \AA)$, there are also cases when $I+V$ and $I-V$ profiles are very different and at great distances from the center, 130$180 \mathrm{~m} \AA$ (Lozitsky, 2009). Perhaps this indicates the existence of even stronger fields in flares.

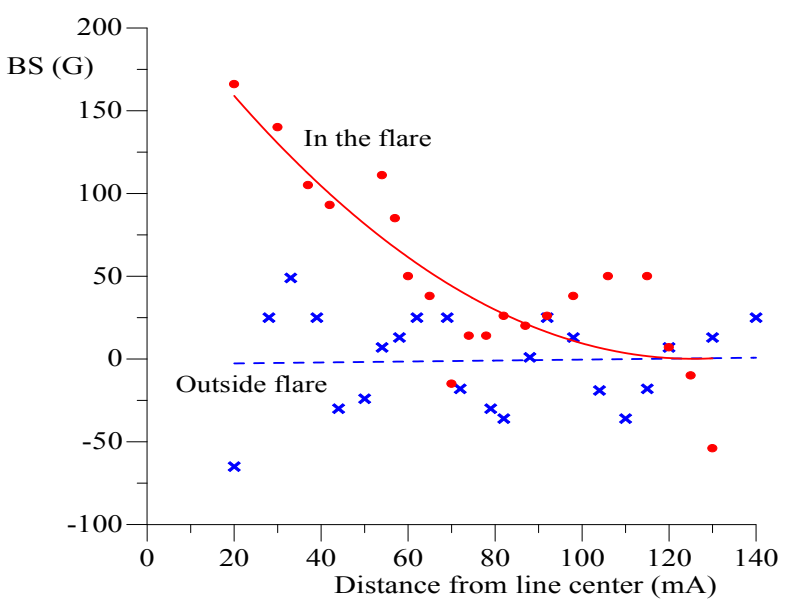

Figure 5: Observational manifestation of the Zeeman effect in core of FeI 5576 line in flare of 5 November 2004: crosses and dashed line - splitting of bisectors of $I \pm V$ profiles in this line, $B S$, formally calibrated to case of FeI 5233 line $\left(\mathrm{g}_{\mathrm{eff}}=1.261\right)$, outside the flare; filled circles and solid line - observed $B S$ splitting in bright knot of flare (Lozitsky and Staude, 2008).

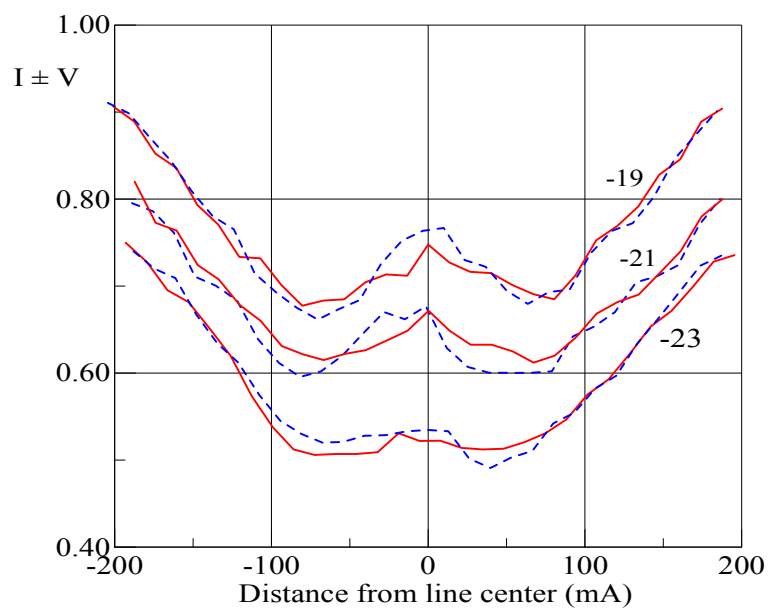

Figure 6: Observed Stokes $I \pm V$ profiles of FeI 5434.5 line in flare of 17 July 2004 (Lozitsky, 2011). The numbers from -17 to -21 correspond to the numbers of the photometric sections; adjacent cuts are located at a distance of $1 \mathrm{Mm}$.

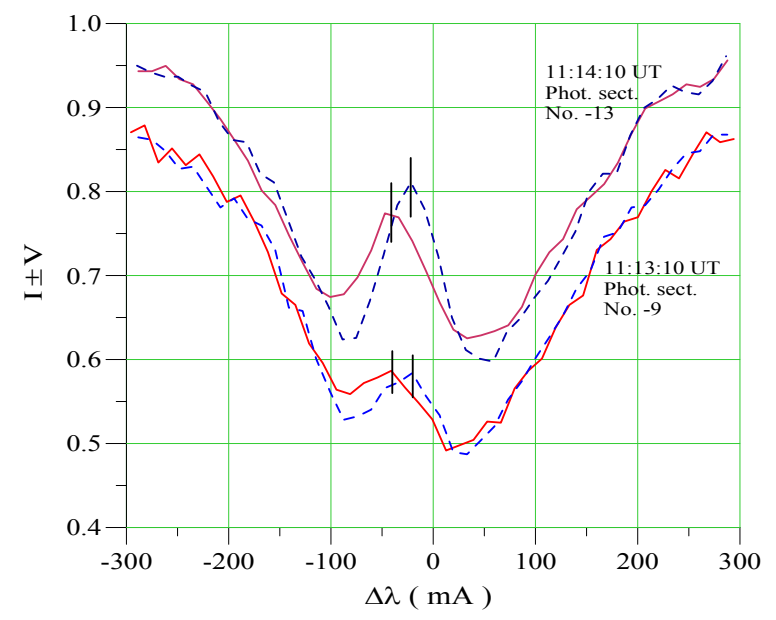

Figure 7: The same as on Fig. 6, but for X17.2/4B flare of 28 October 2003 (Lozitsky and Lozitska, 2017). The spectral splitting of the emission peaks, marked by vertical dashes, corresponds to a magnetic field of about $50 \mathrm{kG}$.

One of the problems of $10^{4} \mathrm{G}$ magnetic fields is that their enormous magnetic pressure exceeds by many orders of magnitude the pressure of the surrounding plasma (Lozitsky, 2015). Such fields should exist either in some special magnetic configurations of the type, perhaps a magnetic vortex (Solov'ev, 2013) or, in general, there may exist a very short time. Concerning the latter, observational data were obtained that the spectral manifestation of these fields exist for at least several minutes (Lozitsky, 1998).

It is highly unlikely that these extremely strong fields form at the same height, where they are observed. Likely, these fields form somewhere in convective zone and they lift on the Sun surface in the ready form. At least in some flares a violet Doppler shift is observed, indicating a lifting of plasma with velocities of about $2 \mathrm{~km} / \mathrm{s}$ (see Fig. 7). 


\section{Semi-empirical models}

The construction of semi-empirical models allows one to increase the spatial resolution in depth and, in addition, makes it possible to obtain altitude profiles of the magnetic field and thermodynamic conditions. This approach requires Stokes-metric data in many spectral lines and also the use of program codes for the calculation of line profiles in a perturbed atmosphere. This gives a lot of important and interesting information about the physical conditions in solar flares. Here we note only one problematic point.

There is no complete clarity in how the magnetic field varies with altitude in solar flares. In some papers, a nonmonotonic change in the magnetic field with altitude has been obtained (see, e.g., Lozitsky et al., 2000), while in others a monotonous attenuation with altitude was obtained (see, e.g. Abramenko and Baranovsky, 2004; Baranovsky et al., 2009).

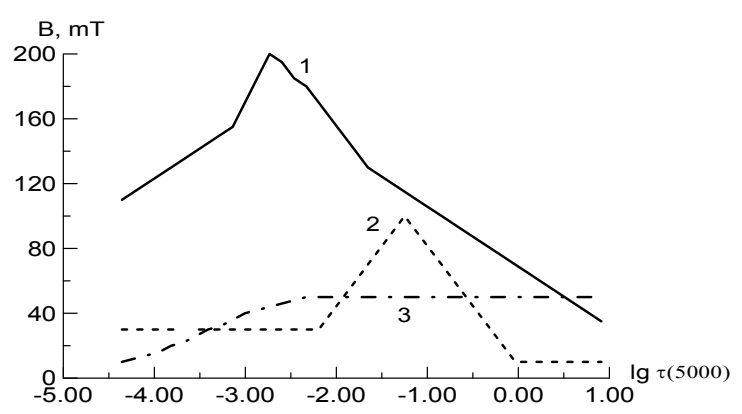

Figure 8: Semi-empirical models of magnetic field for M4.1/1B flare of 5 November 2004 for three moments: 1 - peak of flare, 2 - 4 min after peak, and 3-10 min after peak (see details in paper by Kurochka et al., 2008).

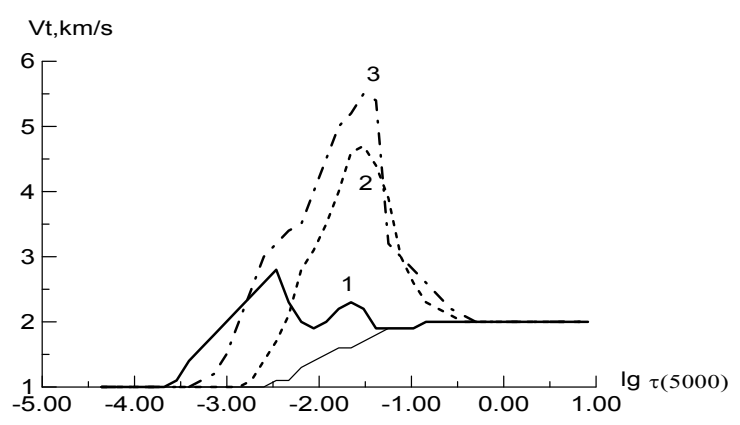

Figure 9: Evolution of the macro-turbulent velocity in M4.1/1B flare of 5 November 2004 (Kurochka et al., 2008).

Perhaps this depends on the phase of the flare development. Thus, in paper by Kurochka et al (2008), a highaltitude peak of the field was obtained at the flare maximum, which gradually "blurred" into the usual monotonous distribution of the magnetic field within 10 minutes (Fig. 8). This issue requires careful study in the future.

It is interesting to note that the macroturbulent velocity changed in the flare on 5 November 2004 differently than the magnetic field (Fig. 9). At maximum phase of the flare this velocity did not exceed $2.5 \mathrm{~km} / \mathrm{s}$, whereas in $10 \mathrm{~min}$ utes it increased to $5.5 \mathrm{~km} / \mathrm{s}$. Apparently, these data di- rectly indicate that the energy of the magnetic field has been transformed into the energy of turbulent motions.

\section{Conclusions}

Our main conclusions are following: (a) upper limit of local magnetic fields in solar flares is, at least, $\sim 10^{4} \mathrm{G}$, (b) such extremely strong fields can occur in very small, spatially unresolved scales, (c) lifetime of such fields is, at least, a few minutes. Probably, the sub-telescopic regions in flares with such very strong fields have a sharply reduced temperature and an increased plasma density. Local non-monotonous distribution of magnetic field strength versus height are possible in flares, their lifetime is also several minutes.

Acknowledgements. This study was funded by the Taras Shevchenko National University of Kyiv, project No. 16БФ023-01, and by Crimea Astrophysical Observatory, project "Physical relationships of solar formations", grant РФФИ № 16-42-910467 p-аэ.

\section{References}

Abramenko V.I., Baranovsky E.A.: 2004, Solar Phys., 220, 81 .

Baranovsky E.A., Lozitsky V.G., Tarashchuk V.P.; 2009, Kinem. Phys. Celest. Bodies, 25, 373.

Botygina O.O., Gordovskyy M.Yu., Lozitsky V.G.: 2016, Adv. Astron. Space Phys., 6, 20.

Gordovskyy M., Lozitsky V.G.: 2014, Solar Phys., 289, 3681.

Kirichek E.A., Solov'ev A.A., Lozitskaya N.I. et al.: 2013, Geomagn. Aeronomy., 53, 831.

Kurochka L.N., Tel'njuk-Adamchuk V.V.: 1978, Solar Phys., 59, 11.

Kurochka E.V., Lozitsky V.G., Osyka O.B.: 2008, Kinem. Phys. Celest. Bodies, 24, 308.

Landi Degl'Innocenti E.L.: 1982, Solar Phys., 77, 285.

Lozitsky V., Masliukh V., Botygina O.: 2015, Bull. Taras Shevch. Nat. Univ. of Kyiv. Astronomy, 52, 7.

Lozitsky V.G., Staude J.: 2008, J. Astrophys. Astron., 29, 387.

Lozitsky V.G.,, Lozitska N.I.: 2017, Bull. Taras Shevch. Nat. Univ. of Kyiv. Astronomy, 55, 51.

Lozitsky V.G.: 1984, PhD Thesis, Kiev, 164p.

Lozitsky V.G.: 1993, Kinem. Phys. Celest. Bodies, 9, 18.

Lozitsky V.G.: 1998, Kinem. Phys. Celest. Bodies, 14, 401.

Lozitsky V.G.: 2009, J. Phys. Studies, 13, 2903-1.

Lozitsky V.G.: 2015, Adv. Space Res., 55, 958.

Lozitsky, V.G., Baranovsky, E.A., Lozitska, N.I. et al.: 2000, Solar Phys. 191, 171.

Lozitsky, V.G.: 2011, Int. J. Astron. Astrophys., 1, 147.

Rachkovsky D.N., Tsap T.T., Lozitsky V.G.: 2005. J. Astrophys. Astron., 26, 435.

Solov'ev A.A..:2013, Solar Phys., 286, 441.

Stenflo J.O.: 1973, Solar Phys., 32, 41.

Stenflo J.O.: 2011, $A \& A$, 529, id.A42, 20 pp.

Varsik J., Plymate C., Goode P et al.: 2014, Proc. of SPIE, 9147, id.91475D 10pp. 